\title{
GENETIC DIVERSITY OF WILD AND CULTIVATED MANGO GENOTYPES OF PAKISTAN USING SSR MARKERS
}

\author{
Rehan Riaz ${ }^{1}$, Ahmad Sattar Khan ${ }^{1, *}, K_{\text {Khurram Ziaf }}{ }^{1}$ and Hafiza Masooma Naseer Cheema ${ }^{2}$ \\ ${ }^{1}$ Institute of Horticultural Sciences, University of Agriculture, Faisalabad-38040, Pakistan; ${ }^{2}$ Plant Genetic Resources \\ Lab, Department of Plant Breeding and Genetics, University of Agriculture, Faisalabad-38040, Pakistan \\ "Corresponding author's e.mail: ahmad_khan157@yahoo.com
}

\begin{abstract}
Pakistan is blessed with a wide range of indigenous mango germplasm. Wild mango genotypes, growing at Azad Jammu and Kashmir (AJK) and its vicinity are valuable resource for unique genetic diversity. The DNA fingerprints of this available germplasm have never been worked out. Hence, the aim of this study was to develop DNA profiles of 31 wild and 13 cultivated genotypes of the country to determine the population structure. Number of alleles per locus of the 51 Simple sequence repeat (SSR) markers ranged from 3 to 9 and a total of 296 alleles with an average of 5.80 alleles per locus. The average polymorphism information content value was 0.764 . The expected and observed heterozygosity values were 0.805 and 0.720 , respectively, which exhibited high level of genetic diversity in the wild and cultivated mango germplasm. The Bayesian cluster, principal coordinate and hierarchical clustering analyses divided the collected genotypes into three groups i.e. A, B and C. Members of group A and B consisted of wild genotypes entirely, while all commercial genotypes were clustered in group C. The obtained results highlighted genetic diversity encompassed by wild mango genotypes of AJK which can be considered as distinct genotypes for further evaluations in the framework of breeding programs and new cultivar identification in mango.
\end{abstract}

Keywords: Tropical fruits, mango cultivars, population structure, genetic resources, germplasm, genetic markers

\section{INTRODUCTION}

Mango (Mangifera indica L.) is one of the most important fruit crops cultivated in tropical and subtropical regions of the world. It is commercially cultivated in about 103 countries of the world. Global production of mango has been estimated to be 40 million tonnes, out of which Pakistan shares $4.5 \%$ (FAOSTAT, 2015). Pakistan is ranked sixth in the world after India, China, Thailand, Indonesia and Mexico (FAOSTAT, 2015). Pakistani mangoes have gained popularity in the world due to their high nutritive value, attractive colour, smooth texture, excellent flavour and fine aroma (Ullah et al., 2012). Being the center of origin and diversity, Indo-Pak subcontinent has broad history (more than 4000 years) of mango cultivation (Ravishankar et al., 2000). There are almost 260 mango varieties reported in Pakistan, among them 'Samar Bahisht Chaunsa', 'Sufaid Chuansa', 'Kala Chaunsa', 'Anwar Ratole', 'Fajri', 'Sindhri', 'Dusehri', 'Faiz Kareem' and 'Langra' are commercially important (Amin and Hanif, 2002). Currently mango cultivars, 'Sindhri', 'Samar Bahisht Chuansa' and 'Sufaid Chaunsa' are being exported to Middle East, Southeast Asia, EU and USA (Nafees et al., 2013). These mango genotypes are heavily threatened by poor orchard management practices, alternate bearing, various diseases, insect pests and physiological disorders, which affects production and export volume (Rajwana et al., 2008). Rich diversity present in indigenous wild germplasm offers a scope to find promising varieties having regular bearing with long shelf-life. But, this indigenous mango germplasm is depleting in their natural habitats and there is a dire need to collect, conserve and utilize this enriched source of diversity. It is imperative for the maintenance of genetic variability, the resistance to genetic erosion (Cunha et al., 2009) and introgression of economically important traits to sustain productivity and survival under changing climatic conditions. Characterization and selection from existing germplasm also offers a plausible way of improving genotypes with desirable traits.

Azad Jammu and Kashmir region of the country is blessed with wide range of indigenous seedling mango germplasm. This germplasm varies in fruit size, fruit colour, bearing habits, flavour, taste, juiciness, ripening time, and texture (Khan et al., 2015; Khan et al., 2016). These seedling mango trees with desirable traits can be served as a novel source for future crop improvement and sustainable mango production (Singh and Jawanda, 1962; Ravishankar et al., 2000). Moreover, wild mango germplasm is not only a source of genetic variation but also possesses tolerance against different pathogens. It can also be used to widen the genetic base of cultivated mango varieties along with offering a scope to extract desirable genes and their utilization in mango improvement and breeding programmes. So, compiling the DNA fingerprints to analyze and document the genetic landmarks can probe the evolutionary relationship between wild and cultivated populations. 
Previously genetic variability among mango varieties has been estimated based on morphological and biochemical characters (Zaied et al., 2007; Rajwana et al., 2011; Begum et al., 2014; Azmat et al., 2016; Khan et al., 2016). These markers have limited features to identify crops and can vary with environment (Karihalo et al., 2003). Microsatellites or SSR are the most common DNA markers which have become the most appropriate and suitable choice for the analysis of genetic diversity and fingerprinting in mango due to their codominance nature, large allelic diversity, reproducibility, polymorphism and amenable to high throughput screening (Viruel et al., 2005; Schnell et al., 2006; Dillon et al., 2013). Wild mango germplasm of the country is still unexplored and has not been properly documented. In addition, its relationship with commercial mango cultivars of the country yet to be studied. So, the present work aims to determine the population structure of 44 wild and cultivated mango genotypes using 55 SSR markers. The objectives of this work were to distinguish the collected mango genotypes, to determine the genetic diversity and relationship among them and to provide useful information for the conservation and utilization of valuable traits in future mango improvement and breeding programme.

\section{MATERIALS AND METHODS}

Plant materials: A total of 44 genotypes (31 wild and 13 cultivated) collected from AJK and adjoining plain areas of Punjab-Pakistan were analyzed in this study. The sampling area lies at an altitude of 200 to $900 \mathrm{~m}$ above the sea level. The topography of sampling area is moderately hilly with valleys and stretches of plains. The climate of AJK ranges from temperate to subtropical, while mango grows mainly in subtropical regions. The average temperature in AJK and Northern Punjab districts ranges from $25^{\circ} \mathrm{C}$ to $35^{\circ} \mathrm{C}$, while in Multan from $25^{\circ} \mathrm{C}$ to $40^{\circ} \mathrm{C}$ in Multan. Sampling of wild germplasm was done from subtropical region of $\mathrm{AJK}$ [Bhimber (22), Kotli (2) and Mirpur (4) districts], and Northern Punjab [Sialkot (2) and Gujrat (1) districts]. Thirteen commercially grown varietal voucher samples were collected from germplasm unit located at Mango Research Station, Shujabad, Multan. The geographical position of each sampled tree was recorded using a hand-held global positioning system (GPS map 76CS X, Garmin, Taipei, Taiwan) along with location information and local names of the surveyed trees (Table 1). Young and tender leaf tissues were collected, washed thoroughly with distilled water, dried, packed in zipper bags and stored at $-80^{\circ} \mathrm{C}$ before DNA extraction.

DNA extraction: Leaf samples were ground to powder form and genomic DNA was extracted following a modified CTAB method (Azmat et al., 2012). DNA concentration and purity were assessed using gel electrophoresis and comparison with Lambda Hind III marker (Fermentas, Vilnius, Lithuania).
DNA samples were subsequently diluted to a working stock with final concentration of $10 \mathrm{ng} \mu \mathrm{L}^{-1}$ and stored at $4^{\circ} \mathrm{C}$.

PCR condition and PCR product analysis: Fifty-five polymorphic SSR markers were initially screened and selected based on PIC values adapted from previously reported studies (Table 2). Forward primers were tagged with fluorescent compounds i.e., FAM or HEX, and PCR was conducted for all 44 samples. PCR reaction mixture $(15 \mu \mathrm{L}$ final volume) contained $10 \mathrm{ng} / \mu \mathrm{L}$ template DNA, $2 \mu \mathrm{L}$ of 10X Taq buffer (pH 8.3), $2.5 \mathrm{mM} \mathrm{MgCl} 2,2.5 \mathrm{mM}$ dNTPs, $1 \mathrm{U}$ of Taq DNA polymerase (MBI, Fermentas, Vilnius, Lithuania) and $10 \mathrm{pmol}$ of each of the forward and reverse primers. SSR markers were amplified by using Bio-Rad C1000 thermocycler (Applied Biosystems, Foster City, CA, USA) with an initial denaturation step of $10 \mathrm{~min}$ at $95^{\circ} \mathrm{C}$, followed by 35 cycles of $45 \mathrm{~s}$ at $92^{\circ} \mathrm{C}, 45 \mathrm{~s}$ at 48 to $56^{\circ} \mathrm{C}$ and $1 \mathrm{~min}$ at $72^{\circ} \mathrm{C}$. The program ended with one additional final extension at $72^{\circ} \mathrm{C}$ for $10 \mathrm{~min}$. PCR amplification conditions for annealing temperature and $\mathrm{MgCl}_{2}$ were optimized for all SSR markers. Fifty-one primers were successfully amplified with desired allele sizes and selected for their high reproducibility. Allele sizes were resolved by using an automated ABI 3130 Genetic analyzer (Applied Biosystems, Foster City, CA, USA). Raw data were analyzed using GeneMapper v4.1 (Applied Biosystems, Foster City, CA, USA) software to score genotypes.

Data analysis: Population genetic parameters for each marker and genotype such as number of alleles per locus (Na), number of effective alleles $(\mathrm{Ne})$, observed heterozygosity $\left(\mathrm{H}_{\mathrm{o}}\right)$, expected heterozygosity $\left(\mathrm{H}_{\mathrm{e}}\right)$, and polymorphic information content (PIC) for each marker locus were estimated using "GenAlEx 6.5" (Peakall and Smouse, 2012). Multilocus matching was also performed to identify duplicates in the data set, using "GenAlEx 6.5" (Peakall and Smouse, 2012). Genotypes with different names but genetically identical at all 51 loci were considered duplicates. Pair-wise genetic distances were computed using the DISTANCE procedure implemented in GenAlEx 6.5 (Peakall and Smouse, 2012).

To assess ability of the markers to infer genetic diversity, distribution and relationship between cultivated and wild genotypes, multivariate approaches i.e. principle coordinate analysis (PCoA), hierarchical clustering (Ward method), were conducted using DARWin6 (Perrier et al., 2006) and Bayesian clustering analysis was determined using STRUCTURE (Pritchard et al., 2000). A burn-in of 500,000 Markov Chain Monte Carlo (MCMC) iterations with a subsequent 250,000 data generating iterations and range of cluster number $(\mathrm{K})$ from one to ten was used with 10 replicates. Evanno's approach (Evanno et al., 2005) was used to determine the most appropriate number of genetic clusters (K). The genetic variation among individuals within and between populations was further investigated through an Analysis of Molecular Variance (AMOVA) using GenAlEx 
Table 1. List of names, collection place, origin and GPS values of 31 wild and 13 cultivated Mangifera indica genotypes.

\begin{tabular}{|c|c|c|c|c|c|c|}
\hline Sr. No. & Genotypes & Area of collection/District & Origin & Longitude (DD) & Latitude (DD) & Elevation \\
\hline & Cultivated & & & & & \\
\hline 1 & Anwar Ratole & MRS, Multan, Punjab & North India & 29.8787 & 71.3490 & $114 \mathrm{M}$ \\
\hline 2 & Dusehri & MRS, Multan, Punjab & North India & 29.8780 & 71.3489 & $115 \mathrm{M}$ \\
\hline 3 & Faiz Kareem & MRS, Multan, Punjab & Multan, Pakistan & 29.8783 & 71.3487 & $117 \mathrm{M}$ \\
\hline 4 & Fajri & MRS, Multan, Punjab & North East India & 29.8782 & 71.3476 & $115 \mathrm{M}$ \\
\hline 5 & Kala Chaunsa & MRS, Multan, Punjab & Multan, Pakistan & 29.8790 & 71.3479 & $116 \mathrm{M}$ \\
\hline 6 & Langra & MRS, Multan, Punjab & North India & 29.8811 & 71.3485 & $114 \mathrm{M}$ \\
\hline 7 & Late Ratole No.12 & MRS, Multan, Punjab & Multan, Pakistan & 29.8824 & 71.3484 & $115 \mathrm{M}$ \\
\hline 8 & Late Ratole No.14 & MRS, Multan, Punjab & Multan, Pakistan & 29.8820 & 71.3480 & $111 \mathrm{M}$ \\
\hline 9 & Neelum & MRS, Multan, Punjab & South India & 29.8827 & 71.3537 & $113 \mathrm{M}$ \\
\hline 10 & Ratole No. 3 & MRS, Multan, Punjab & India & 29.8781 & 71.3494 & $111 \mathrm{M}$ \\
\hline 11 & Samar Bahisht Chaunsa & MRS, Multan, Punjab & North India & 29.8823 & 71.3483 & $114 \mathrm{M}$ \\
\hline 12 & Sindhri & MRS, Multan, Punjab & Sindh, Pakistan & 29.8822 & 71.3530 & $115 \mathrm{M}$ \\
\hline \multirow[t]{2}{*}{13} & Sufaid Chaunsa & MRS, Multan, Punjab & Multan, Pakistan & 29.8819 & 71.3520 & $112 \mathrm{M}$ \\
\hline & Wild types & & & & & \\
\hline 14 & BMB-38 & Bhimber, AJK & Pakistan & 33.0506 & 74.0424 & $610 \mathrm{M}$ \\
\hline 15 & BMB-39 & Bhimber, AJK & Pakistan & 33.1326 & 74.0435 & $618 \mathrm{M}$ \\
\hline 16 & BMB-61 & Bhimber, AJK & Pakistan & 32.9317 & 74.0260 & $314 \mathrm{M}$ \\
\hline 17 & BMB-78 & Bhimber, AJK & Pakistan & 32.8929 & 74.2817 & $384 \mathrm{M}$ \\
\hline 18 & BMB-80 & Bhimber, AJK & Pakistan & 32.8993 & 74.2843 & $387 \mathrm{M}$ \\
\hline 19 & BMB-92 & Bhimber, AJK & Pakistan & 32.9061 & 74.2168 & $450 \mathrm{M}$ \\
\hline 20 & BMB-119 & Bhimber, AJK & Pakistan & 32.8765 & 74.2868 & $345 \mathrm{M}$ \\
\hline 21 & BMB-134 & Bhimber, AJK & Pakistan & 32.9068 & 74.2882 & $456 \mathrm{M}$ \\
\hline 22 & BMB-135 & Bhimber, AJK & Pakistan & 32.9069 & 74.2882 & $454 \mathrm{M}$ \\
\hline 23 & BMB-137 & Bhimber, AJK & Pakistan & 32.9075 & 74.2889 & $467 \mathrm{M}$ \\
\hline 24 & BMB-138 & Bhimber, AJK & Pakistan & 32.9070 & 74.2884 & $463 \mathrm{M}$ \\
\hline 25 & BMB-177 & Bhimber, AJK & Pakistan & 32.9095 & 74.2175 & $379 \mathrm{M}$ \\
\hline 26 & BMB-179 & Bhimber, AJK & Pakistan & 32.9089 & 74.2168 & $378 \mathrm{M}$ \\
\hline 27 & BMB-180 & Bhimber, AJK & Pakistan & 32.9096 & 74.2174 & $378 \mathrm{M}$ \\
\hline 28 & BMB-213 & Bhimber, AJK & Pakistan & 32.9361 & 74.0240 & $314 \mathrm{M}$ \\
\hline 29 & BMB-214 & Bhimber, AJK & Pakistan & 32.9354 & 74.0237 & $317 \mathrm{M}$ \\
\hline 30 & BMB-215 & Bhimber, AJK & Pakistan & 32.9353 & 74.0234 & $317 \mathrm{M}$ \\
\hline 31 & BMB-216 & Bhimber, AJK & Pakistan & 32.9343 & 74.0262 & $309 \mathrm{M}$ \\
\hline 32 & BMB-219 & Bhimber, AJK & Pakistan & 33.0389 & 74.2331 & $832 \mathrm{M}$ \\
\hline 33 & BMB-220 & Bhimber, AJK & Pakistan & 33.0384 & 74.2040 & $832 \mathrm{M}$ \\
\hline 34 & BMB-222 & Bhimber, AJK & Pakistan & 33.0430 & 74.1971 & $849 \mathrm{M}$ \\
\hline 35 & BMB-227 & Bhimber, AJK & Pakistan & 33.0411 & 74.1986 & $901 \mathrm{M}$ \\
\hline 36 & GRT-185 & Gujrat, Punjab & Pakistan & 32.6968 & 74.3271 & $174 \mathrm{M}$ \\
\hline 37 & KTL-19 & Kotli, AJK & Pakistan & 33.3800 & 73.8768 & $545 \mathrm{M}$ \\
\hline 38 & KTL-27 & Kotli, AJK & Pakistan & 33.3800 & 73.8768 & $722 \mathrm{M}$ \\
\hline 39 & MRP-02 & Mirpur, AJK & Pakistan & 32.4113 & 73.6262 & $559 \mathrm{M}$ \\
\hline 40 & MRP-03 & Mirpur, AJK & Pakistan & 32.4109 & 73.6258 & $565 \mathrm{M}$ \\
\hline 41 & MRP-07 & Mirpur, AJK & Pakistan & 33.4104 & 73.6275 & $541 \mathrm{M}$ \\
\hline 42 & MRP-14 & Mirpur, AJK & Pakistan & 33.3710 & 73.6604 & $499 \mathrm{M}$ \\
\hline 43 & SKT-203 & Sialkot, Punjab & Pakistan & 32.6499 & 74.4831 & $243 \mathrm{M}$ \\
\hline 44 & SKT-211 & Sialkot, Punjab & Pakistan & 32.6440 & 74.4835 & $245 \mathrm{M}$ \\
\hline
\end{tabular}

Code: Azad Jammu and Kashmir (AJK), Bhimber (BMB), Gujrat (GRT), Kotli (KTL), Mirpur (MRP), Mango Research Station (MRS), and Sialkot (SKT)

6.5 (Peakall and Smouse, 2012). Groups were defined according to clusters obtained by the Bayesian analysis.

\section{RESULTS}

Results indicated that out of 55 SSR markers used to evaluate the genetic diversity in wild and cultivated mango genotypes, 51 showed consistent high quality amplification, while three markers failed to amplify and one was monomorphic. In the subsequent screening, 44 genotypes were screened using 51 selected SSR markers (Table 3). A total of 296 alleles were found with size ranging from 99 to $344 \mathrm{bp}$. The number of 
alleles ranged from 3 (mMiCIR001) to 9 (MillHR-34) with an average of 5.80 alleles per locus. The observed heterozygosity $\left(\mathrm{H}_{0}\right)$ ranged from 0.364 (LMMA9) to 0.864 (MillHR-34) with a mean of 0.715 .

Table 3. Salient characteristics of 51 SSR markers used for genotyping.

\begin{tabular}{|c|c|c|c|c|c|c|}
\hline Sr. & Locus & $\mathbf{N a}$ & $\mathrm{Ne}$ & Ho & $\mathrm{He}$ & PIC \\
\hline 1 & MiSHRS-1 & 6 & 24 & 0.744 & 0.779 & 0.738 \\
\hline 2 & MiSHRS-32 & 4 & 14 & 0.523 & 0.720 & 0.665 \\
\hline 3 & LMMA9 & 4 & 24 & 0.364 & 0.528 & 0.496 \\
\hline 4 & LMMA15 & 5 & 18 & 0.545 & 0.614 & 0.539 \\
\hline 5 & MIAC5 & 6 & 33 & 0.750 & 0.792 & 0.755 \\
\hline 6 & mMiCIR001 & 3 & 11 & 0.548 & 0.781 & 0.541 \\
\hline 7 & mMiCIR003 & 4 & 18 & 0.748 & 0.830 & 0.796 \\
\hline 8 & mMiCIR008 & 7 & 2 & 0.818 & 0.900 & 0.881 \\
\hline 9 & mMiCIR009 & 8 & 26 & 0.784 & 0.896 & 0.876 \\
\hline 10 & mMiCIR013 & 6 & 24 & 0.484 & 0.863 & 0.836 \\
\hline 11 & mMiCIR016 & 6 & 34 & 0.649 & 0.849 & 0.819 \\
\hline 12 & mMiCIR018 & 7 & 26 & 0.789 & 0.835 & 0.804 \\
\hline 13 & mMiCIR021 & 5 & 22 & 0.830 & 0.874 & 0.848 \\
\hline 14 & mMiCIR022 & 7 & 29 & 0.841 & 0.892 & 0.871 \\
\hline 15 & mMiCIR025 & 4 & 12 & 0.650 & 0.796 & 0.754 \\
\hline 16 & mMiCIR028 & 7 & 48 & 0.682 & 0.806 & 0.767 \\
\hline 17 & mMiCIR029 & 8 & 42 & 0.837 & 0.865 & 0.839 \\
\hline 18 & mMiCIR032 & 6 & 26 & 0.852 & 0.870 & 0.845 \\
\hline 19 & mMiCIR034 & 6 & 19 & 0.760 & 0.789 & 0.744 \\
\hline 20 & mMiCIR036 & 5 & 17 & 0.682 & 0.748 & 0.711 \\
\hline 21 & MiIIHR01 & 8 & 38 & 0.442 & 0.761 & 0.724 \\
\hline 22 & MiIIHR03 & 7 & 19 & 0.659 & 0.820 & 0.786 \\
\hline 23 & MiIIHR05 & 4 & 18 & 0.731 & 0.776 & 0.734 \\
\hline 24 & MiIIHR06 & 4 & 12 & 0.614 & 0.705 & 0.649 \\
\hline 25 & MiIIHR07 & 5 & 16 & 0.808 & 0.812 & 0.774 \\
\hline 26 & MiIIHR09 & 5 & 28 & 0.721 & 0.800 & 0.757 \\
\hline 27 & MiIIHR10 & 6 & 34 & 0.750 & 0.783 & 0.741 \\
\hline 28 & MiIIHR12 & 5 & 1 & 0.523 & 0.748 & 0.696 \\
\hline 29 & MiIIHR13 & 5 & 21 & 0.682 & 0.759 & 0.713 \\
\hline 30 & MiIIHR14 & 4 & 15 & 0.659 & 0.744 & 0.686 \\
\hline 31 & MiIIHR16 & 5 & 22 & 0.636 & 0.786 & 0.741 \\
\hline 32 & MiIIHR17 & 6 & 24 & 0.659 & 0.750 & 0.699 \\
\hline 33 & MiIIHR18 & 5 & 29 & 0.684 & 0.792 & 0.749 \\
\hline 34 & MiIIHR19 & 5 & 15 & 0.784 & 0.806 & 0.770 \\
\hline 35 & MiIIHR20 & 8 & 52 & 0.848 & 0.852 & 0.822 \\
\hline 36 & MiIIHR21 & 6 & 38 & 0.717 & 0.803 & 0.761 \\
\hline 37 & MillHR22 & 7 & 27 & 0.814 & 0.834 & 0.801 \\
\hline 38 & MiIIHR23 & 4 & 11 & 0.697 & 0.780 & 0.735 \\
\hline 39 & MiIIHR24 & 6 & 32 & 0.750 & 0.806 & 0.769 \\
\hline 40 & MiIIHR25 & 8 & 27 & 0.818 & 0.886 & 0.864 \\
\hline 41 & MiIIHR26 & 6 & 17 & 0.773 & 0.807 & 0.767 \\
\hline 42 & MiIIHR27 & 6 & 23 & 0.785 & 0.792 & 0.748 \\
\hline 43 & MiIIHR28 & 5 & 21 & 0.731 & 0.751 & 0.699 \\
\hline 44 & MiIIHR29 & 5 & 18 & 0.773 & 0.829 & 0.794 \\
\hline 45 & MiIIHR30 & 5 & 12 & 0.748 & 0.776 & 0.730 \\
\hline 46 & MiIIHR31 & 8 & 24 & 0.750 & 0.868 & 0.844 \\
\hline 47 & MiIIHR32 & 5 & 32 & 0.682 & 0.804 & 0.766 \\
\hline 48 & MiIIHR33 & 7 & 18 & 0.830 & 0.885 & 0.862 \\
\hline 49 & MiIIHR34 & 9 & 52 & 0.864 & 0.903 & 0.883 \\
\hline 50 & MiIIHR35 & 8 & 34 & 0.841 & 0.875 & 0.851 \\
\hline 51 & MiIIHR36 & 5 & 14 & 0.832 & 0.867 & 0.840 \\
\hline
\end{tabular}

The expected heterozygosity $(\mathrm{He})$ varied from 0.528 for LMMA9 to 0.903 for MillHR-34, with a mean of 0.803. All SSR markers were highly polymorphic which displayed maximum PIC value 0.883 at locus (MillHR-34), while minimum PIC value 0.496 at locus LMMA9, with a mean of 0.762. Pairwise comparisons of individual genotypes did not identify any matching genotypes, which indicates absence of mislabelling or duplicates in our collected mango genotypes. The principle coordinate analysis (PCoA), hierarchical clustering (Ward method) and a model-based clustering method implemented in the program STRUCTURE clearly grouped all genotypes into three distinct groups with each group containing same set of genotypes. PCoA showed spatial distribution among genotypes into three distinct groups (Fig. 1). The maximum dissimilarity was observed in wild genotypes of group A and B. While, the minimum dissimilarity was observed in group $\mathrm{C}$ which mainly occupied commercial mango genotypes. The axis 1 and 2 represent 16.57 and $12.25 \%$ variation, respectively.

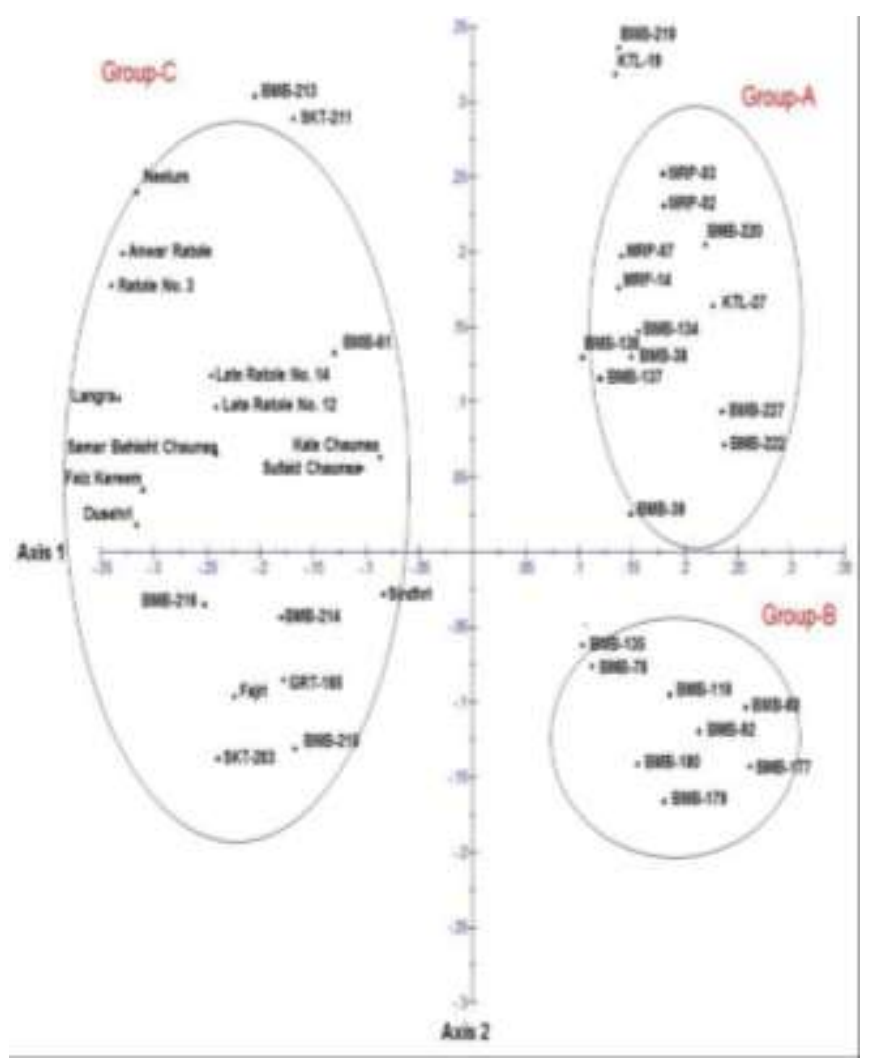

Figure 1.Principle coordinates analysis of 44 mango genotypes from AJK and Punjab with 51 SSR markers.

The Ward and UPGMA hierarchical clustering methods were used to differentiate the wild genotypes from cultivated varieties (Fig. 2). Group-A consisted of wild type genotypes from high to mid altitude areas (450-900 m) in the AJK, which 
was comprised of two sub-groups. Nine of mango genotypes, namely, 'BMB-137', 'BMB-138', 'BMB-134', 'BMB-38', 'KTL-27', 'MRP-14', 'MRP-07', 'MRP-03' and 'MRP-02', which are wild types, were in subgroup one. Other six wild types ('BMB-227', 'BMB-222', 'BMB-220', 'BMB-39', 'BMB-219', and 'KTL-19') were grouped together. Group-B contained eight wild genotypes viz., 'BMB-92', 'BMB-135', 'BMB-119', 'BMB-180', 'BMB-78', 'BMB-179', 'BMB177 ', and 'BMB-80', from low hill regions (340-450 m) of AJK. Group-C consisted of 14 cultivated mango varieties with eight wild type genotypes of $M$. indica from northern Punjab. Indian cultivars 'Neelum' and 'Anwar Ratole' were close to each other in all three analyses. 'Samar Bahisht Chaunsa' was close to 'Faiz Kareem' in all three analyses, because former is considered as one of the parents of 'Faiz Kareem' (Rajwana et al., 2010). The cluster analysis indicated that genotypes grouping was according to origin and the nature type was only at major group level and not along the sub-group level that indicates significant variations/differences among the collected genotypes.

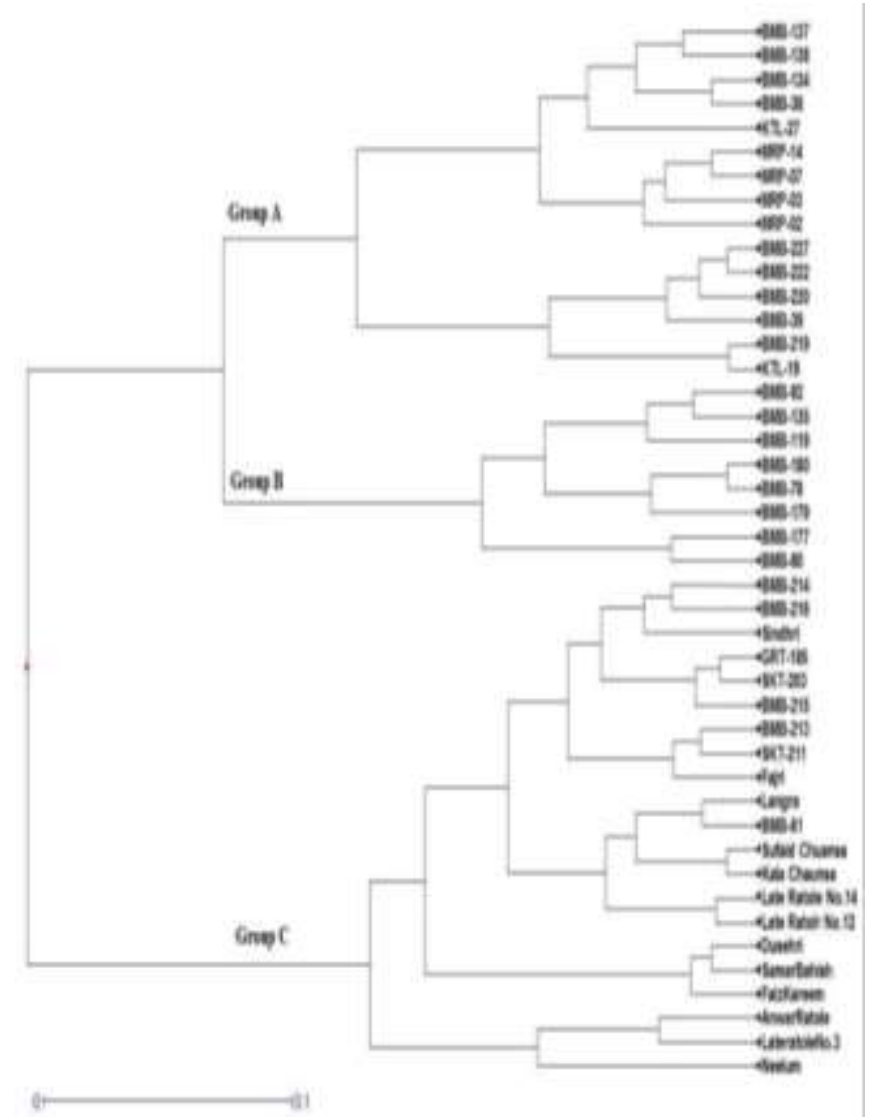

Figure 2. Dendrogram of 44 genotypes based on hierarchal cluster analysis (Ward method) using the simple dissimilarity matrix derived from $\mathbf{5 1}$ SSR markers.
The highest likelihood value was obtained maximum when the number of sub-populations $(\mathrm{K})=3$ (Fig. 3). The three groups produced by STRUCTURE were similar to those determined by PCoA. Group A contained 15 wild mango genotypes, all of which originated from high to mid altitude areas of AJK. The Q-value for membership in this group was 0.80 or above for 15 genotypes. Q-values of 'Langra', 'BMB215 ' and 'BMB-216' split between the group A and B. The wild genotype 'BMB-214', collected from AJK, had Q-value split between group A and C. Group B consisted of 8 wild genotypes collected from low hilly regions of AJK and were placed in this group with Q-values 0.80 or more. Group C contained all the cultivated varieties with some wild genotypes originated from areas of northern Punjab. A cultivated variety 'Neelum' originated from India had Qvalue split between the group $\mathrm{B}$ and $\mathrm{C}$. The genotypes 'Neelum', 'Langra', 'BMB-214', 'BMB-215', and 'BMB216' having split Q-values between two groups were categorized as admixed ancestry. The analysis showed high level of variation in wild genotypes and maximum genetic similarity in cultivated varieties.

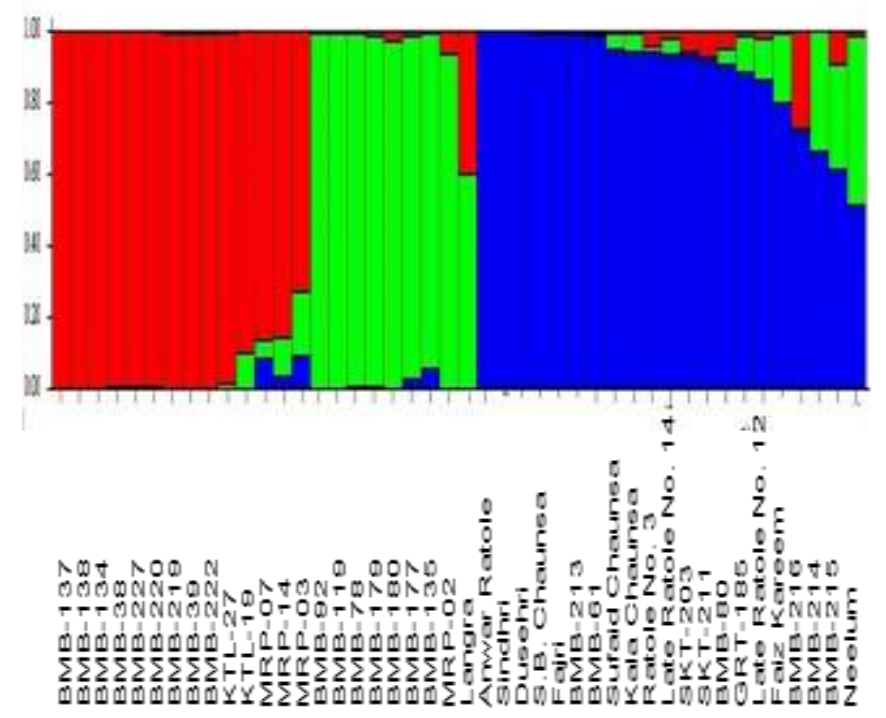

Figure 3. Estimated genetic structure of the wild and cultivated populations based on STRUCTURE analysis at $K=3$.

Analysis of Molecular Variance (AMOVA) showed significant genetic differences among populations which accounted for $7 \%$, whereas $19 \%$ variability was recorded among individuals within a population. Without considering the population boundaries, genetic variation between individual genotypes was highly significant i.e. $74 \%$ (Table 4). The Fst value between wild and cultivated genotypes was $0.069(P=0.001)$. The obtained pairwise Fst value between the populations was $0.050(P=0.000)$. 
Table 4. AMOVA results showing the partitioning of genetic diversity among the wild and cultivated mango genotypes in Pakistan.

\begin{tabular}{lccrrrr}
\hline Source & df & SS & MS & Est. Var. & \% & p-value \\
\hline Among Population & 1 & 21385 & 21385 & 408 & $7 \%$ & $<0.001$ \\
Among Individual & 42 & 278150 & 6623 & 1108 & $19 \%$ & $<0.001$ \\
Within Individual & 44 & 191681 & 4356 & 4356 & $74 \%$ & $<0.001$ \\
Total & 87 & 491216 & & 5831 & $100 \%$ & \\
\hline
\end{tabular}

Table 2. List of forward and reverse primer sequences for 55 SSR used for studying genetic diversity in wild and cultivated mango genotypes.

\begin{tabular}{|c|c|c|c|}
\hline Primer & Sequence 5'-3' & Allele size range & Reference \\
\hline $\begin{array}{l}\text { MiSHRS-1F } \\
\end{array}$ & TAACAGCTTTGCTTGCCTCC & $189-216$ & Schnell et al. (2005) \\
\hline MiSHRS-1R & TCCGCCGATAAACATCAGAC & & \\
\hline MiSHRS-32F & TTGATGCAACTTTCTGCC & $190-203$ & Schnell et al. (2005) \\
\hline MiSHRS-32R & ATGTGATTGTTAGAATGAACTT & & \\
\hline LMMA9-F & TTGCAACTGATAACAAATATAG & 174-184 & Viruel et al. (2005) \\
\hline LMMA9-R & TTCACATGACAGATATACACTT & & \\
\hline LMMA15-F & AACTACTGTGGCTGACATAT & 207-219 & Viruel et al. (2005) \\
\hline LMMA15-R & CTGATTAACATAATGACCATCT & & \\
\hline MIAC-5F & AATTATCCTATCCCTCGTATC & $118-228$ & Honsho et al. (2005) \\
\hline AB190348-R & AGAAACATGATGTGAACC & & \\
\hline mMiCIR001-F & TGAGTTGTTGTCCTGCT & $191-203$ & Duval et al. (2005) \\
\hline mMiCIR001-R & GGTGCTTGTTTCTCGT & & \\
\hline mMiCIR003-F & GATGAAACCAAAGAAGTCA & $306-322$ & Duval et al. (2005) \\
\hline mMiCIR003-R & CCAATAAGAACTCCAACC & & \\
\hline mMiCIR008-F & GACCCAACAAATCCAA & $156-184$ & Duval et al. (2005) \\
\hline mMiCIR008-R & ACTGTGCAAACCAAAAG & & \\
\hline mMiCIR009-F & AAAGATAAGATTGGGAAGAG & $151-170$ & Duval et al. (2005) \\
\hline mMiCIR009-R & CGTAAGAAGAGCAAAGGT & & \\
\hline mMiCIR013-F & GCGTAAAGCTGTTGACTA & $144-160$ & Duval et al. (2005) \\
\hline mMiCIR013-R & TCATCTCCCTCAGAACA & & \\
\hline mMiCIR016-F & TAGCTGTTTTGGCCTT & $228-246$ & Duval et al. (2005) \\
\hline mMiCIR016-R & ATGTGGTTTGTTGCTTC & & \\
\hline mMiCIR018-F & ССТCAАТСТСАСТСААСА & 202-244 & Duval et al. (2005) \\
\hline mMiCIR018-R & ACCССАCAАТCAАACTAC & & \\
\hline mMiCIR021-F & ССАТТСТССАТССААА & $162-184$ & Duval et al. (2005) \\
\hline mMiCIR021-R & TGCATAGCAGAAAGAAGA & & \\
\hline mMiCIR022-F & TGTCTACCATCAAGTTCG & $145-172$ & Duval et al. (2005) \\
\hline mMiCIR022-R & GCTGTTGTTGCTTTACTG & & \\
\hline mMiCIR025-F & ATCCCCAGTAGCTTTGT & $212-230$ & Duval et al. (2005) \\
\hline mMiCIR025-R & TGAGAGTTGGCAGTGTT & & \\
\hline mMiCIR028-F & AAGAGGGAATCTTAATCAAC & 175-197 & Duval et al. (2005) \\
\hline mMiCIR028-R & GTCGTTTTGCGTTAGTG & & \\
\hline mMiCIR029-F & GCGTGTCAATCTAGTGG & $152-202$ & Duval et al. (2005) \\
\hline mMiCIR029-R & GCTTTGGTAAAAGGATAAG & & \\
\hline mMiCIR032-F & TCATTGCTGTCCCTTTTC & $118-172$ & Duval et al. (2005) \\
\hline mMiCIR032-R & ATCGCTCAAACAATCC & & \\
\hline mMiCIR034-F & TCGGTCATTTACACCTCT & 192-216 & Duval et al. (2005) \\
\hline mMiCIR034-R & TTATTGAGCTTCTTTGTGTT & & \\
\hline mMiCIR036-F & ACCACGAAAAGACAACTC & $248-272$ & Duval et al. (2005) \\
\hline mMiCIR036-R & TCATCTTTGTTAAATAGGTTAAT & & \\
\hline MiIIHR01-F & GGATGCACAACAACAAGCAC & $237-269$ & Ravishankar et al. (2011) \\
\hline MiIIHR01-R & TCAGCAAGCAATCCCTTCTT & & \\
\hline MiIIHR03-F & GTCGATGCCTGGAATGAAGT & $223-243$ & Ravishankar et al. (2011) \\
\hline MiIIHR03-R & AAGCATCGAACAGCTCCAAT & & \\
\hline MiIIHR05-F & СТСТСССТCACTTGCTCCAC & 181-197 & Ravishankar et al. (2011) \\
\hline MillHR05-R & AGACCACCGACAACGAAAAC & & \\
\hline
\end{tabular}




\begin{tabular}{|c|c|c|c|}
\hline Primer & Sequence 5'-3' & Allele size range & Reference \\
\hline MiIIHR06-F & CGCCGAGCCTATAACCTCTA & $99-113$ & Ravishankar et al. (2011) \\
\hline MiIIHR06-R & ATCATGCCCTAAACGACGAC & & \\
\hline MiIIHR07-F & GCCACTCAGCTAAATAGCCTCT & $153-177$ & Ravishankar et al. (2011) \\
\hline MiIIHR07-R & TGCAGTCGGTAAAGTGATGG & & \\
\hline MiIIHR09-F & GTTGTGACCGAGGCCTTAAA & $273-281$ & Ravishankar et al. (2011) \\
\hline MiIIHR09-R & CTTTGACATCGCTGATCTGG & & \\
\hline MiIIHR10-F & CGATTCAAGACGGAAAGGAA & $163-179$ & Ravishankar et al. (2011) \\
\hline MiIIHR10-R & TTCAAGCACAGACGACCAAC & & \\
\hline MiIIHR12-F & GCCCCATCAATACGATTGTC & $161-173$ & Ravishankar et al. (2011) \\
\hline MiIIHR12-R & ATTTCCCACCATTGTCGTTG & & \\
\hline MiIIHR13-F & CCCAGTTCCAACATCATCAG & $171-185$ & Ravishankar et al. (2011) \\
\hline MiIIHR13-R & TТССТСТGGAAGAGGGAAGA & & \\
\hline MiIIHR14-F & CCGAAACAACTCTTCCTCCA & $332-344$ & Ravishankar et al. (2011) \\
\hline MiIIHR14-R & TGCTCTCTGGCCTCTTCTTC & & \\
\hline MiIIHR16-F & TTTCACTTGGTTCTGGATTGC & $178-186$ & Ravishankar et al. (2011) \\
\hline MiIIHR16-R & ATTTCCCACCATTGTCGTTG & & \\
\hline MiIIHR17-F & GCTTGCTTCCAACTGAGACC & $234-242$ & Ravishankar et al. (2011) \\
\hline MiIIHR17-R & GCAAAATGCTCGGAGAAGAC & & \\
\hline MiIIHR18-F & TCTGACGTCACCTCCTTTCA & $156-164$ & Ravishankar et al. (2011) \\
\hline MiIIHR18-R & ATACTCGTGCCTCGTCCTGT & & \\
\hline MiIIHR19-F & TGATATTTTCAGGGCCCAAG & $181-191$ & Ravishankar et al. (2011) \\
\hline MiIIHR19-R & AAATGGCACAAGTGGGAAAG & & \\
\hline MiIIHR20-F & CCTAACGCGCAAGAAACATA & $176-190$ & Ravishankar et al. (2011) \\
\hline MiIIHR20-R & ACCCACСТTCCCAАТСТTTT & & \\
\hline MiIIHR21-F & TTTGGCTGGGTGATTTTAGC & $225-239$ & Ravishankar et al. (2011) \\
\hline MiIIHR21-R & TTAATTGCAGGACTGGAGCA & & \\
\hline MiIIHR22-F & TGGCCGAACTAGCAAACTCT & $216-228$ & Ravishankar et al. (2011) \\
\hline MiIIHR22-R & CCCCATTTCGAGAAAATTCC & & \\
\hline MiIIHR23-F & TCTGACCCAACAAAGAACCA & $136-144$ & Ravishankar et al. (2011) \\
\hline MiIIHR23-R & TCCTCCTCGTCCTCATCATC & & \\
\hline MiIIHR24-F & GCTCAACGAACCCAACTGAT & $238-248$ & Ravishankar et al. (2011) \\
\hline MiIIHR24-R & TCCAGCATTCAATGAAGAAGTT & & \\
\hline MiIIHR25-F & TGTGAGTCTCCGTTTGTGCT & $143-169$ & Ravishankar et al. (2011) \\
\hline MiIIHR25-R & СССТСТCATTTTCCCAGTCA & & \\
\hline MiIIHR26-F & GCGAAAGAGGAGAGTGCAAG & $136-146$ & Ravishankar et al. (2011) \\
\hline MiIIHR26-R & TCTATAAGTGCCCCCTCACG & & \\
\hline MiIIHR27-F & TGGGGATTCATCGGAGATAG & $186-196$ & Ravishankar et al. (2011) \\
\hline MiIIHR27-R & TGGAAGACCCATTCTCATGC & & \\
\hline MiIIHR28-F & GCGGTCGCAGACAAATTCTATAT & $102-110$ & Ravishankar et al. (2011) \\
\hline MiIIHR28-R & ACAACTCGAGATTGTCACATCTTT & & \\
\hline MiIIHR29-F & CGATGAGGATGGTTGGTTTT & $141-155$ & Ravishankar et al. (2011) \\
\hline MiIIHR29-R & CATCAACAGTCGCCATCAAT & & \\
\hline MiIIHR30-F & AGCTATCGCCACAGCAAATC & $186-194$ & Ravishankar et al. (2011) \\
\hline MiIIHR30-R & GTCTTCTTCTGGCTGCCAAC & & \\
\hline MiIIHR31-F & TTCTGTTAGTGGCGGTGTTG & $215-233$ & Ravishankar et al. (2011) \\
\hline MiIIHR31-R & САССТССТССТССТССТСТТ & & \\
\hline MiIIHR32-F & TGGTGGTGTTTGTTTGCAGT & $172-184$ & Ravishankar et al. (2011) \\
\hline MiIIHR32-R & ACCACCCGCAGTATTGAAAG & & \\
\hline MiIIHR33-F & GAAGCACTTGTCTCCCTTGC & $162-184$ & Ravishankar et al. (2011) \\
\hline MiIIHR33-R & ССТCACACTCСТCСАССТGT & & \\
\hline MiIIHR34-F & CTGAGTTTGGCAAGGGAGAG & $223-251$ & Ravishankar et al. (2011) \\
\hline MiIIHR34-R & TTGATCCTTCACCACCATCA & & \\
\hline MiIIHR35-F & TGGTGAAGCTTGTTGTCTGC & $189-219$ & Ravishankar et al. (2011) \\
\hline MiIIHR35-R & TGGCTTGACTGTTTTTCAGC & & \\
\hline MiIIHR36-F & TCTATAAGTGCCCCCTCACG & $219-241$ & Ravishankar et al. (2011) \\
\hline MiIIHR36-R & ACTGCCACCGTGGAAAGTAG & & \\
\hline
\end{tabular}




\section{DISCUSSION}

It is important to understand the amount and structure of genetic variability present in mango germplasm for conservation, management and further improvement for various important characteristics. It was noticed that surveyed area was an enriched reservoir of genetically diversified wild mango populations. It is a well-established fact that the cultivated genotypes of mango in Indo-Pak subcontinent were mainly selected from naturally occurring chance seedlings and these selected genetic variants have been conserved and subsequently maintained through vegetative propagation. The molecular analysis revealed that cultivated and wild mango germplasm of Pakistan is genetically diverse. The wild mango germplasm has distinct genetic profile. However, it is important to further study the results of this genetic analysis for pedigree analysis and comparison with world mango germplasm to draw the conclusion.

Microsatellite markers have been used widely in Mangifera species for genetic mapping, genetic variation, cultivar identification and phylogenetic analysis for the improvement of mango genotypes (Dillon et al., 2013; Schnell et al., 2006; Viruel et al., 2005). There is enormous and valuable genetic diversity present among Pakistani mango cultivars due to diverse geo-ecological regions and climatic conditions. The present study revealed abundant allelic variation among 51 SSR loci in the characterization of 44 mango genotypes. These markers painted high level of genetic variation by producing a total of 296 alleles. This is lower in comparison to 318 bands detected in 90 mango genotypes with 106 SSR loci by Surapaneni et al. (2013) and higher than the 103 alleles generated in 241 mango genotypes (Singh et al., 2009). The existence of different SSR alleles showed genetic polymorphism in the studied mango genotypes. Moreover, the detection of specific alleles in some genotypes shows the occurrence of deletions/insertions in the DNA. The results for average number of alleles per locus (5.92) fall within the range of previously reported for microsatellite studies of different mango germplasm (Schnell et al., 2006; Viruel et al., 2005). A comparable study of diversity in Indian collection of 387 mango genotypes was carried out by Ravishankar et al. (2015), which revealed a mean of 24.61 alleles per SSR locus and a mean Ho of 0.624 . Another study showed that a panel of 90 Indian local genotypes harboured 2.87 alleles per SSR locus and a mean Ho of 0.29 (Surapaneni et al., 2013). Dillon et al. (2013) reported an average of 12.09 alleles per SSR locus and a mean Ho of 0.69 in Australian National Mango Genebank, which mainly comprised of $M$. indica and its related species. The high level of heterozygosity in our study was attributed to cross pollination and out crossing. Different levels of genetic diversity and polymorphism have been reported in mango using various types of markers, like $90 \%$ and above by SSR markers (Dillon et al., 2013; Surapaneni et al., 2013), 85 to $99 \%$ with ISSR (Ariffin et al., 2015; Samal et al., 2012; Tomar et al., 2011), 73 to $100 \%$ with RAPD (Ravishankar et al., 2000; Karihaloo et al., 2003; Rahman et al., 2007; Rajwana et al., 2008; Souza et al., 2011), 84 to $96 \%$ by AFLP (Yamanaka et al., 2006; Ga'lvez-Lo'pez et al., 2010) and $73 \%$ by SCoT markers (Luo et al., 2010).

The average PIC value obtained in this study was 0.764 which was higher than the average value reported by earlier studies (Surapaneni et al., 2013; Dillon et al., 2013; Ravishankar et al., 2011; Hirano et al., 2010 and Schnell et al., 2005). The markers having higher PIC values are considered highly prospective to reveal allelic variation. The average PIC value of microsatellites generated in different studies varies during the testing of different number of genotypes and microsatellites. Microsatellites are more reliable and due to very high reproducibility it can be successfully used for the cultivar identification by using variety specific primers. The unique allelic pattern was observed mostly in wild genotypes along with two cultivated varieties 'Langra' and 'Neelum', which revealed the presence of unique genetic makeup in these genotypes. This information can be used as molecular signatures in fingerprinting studies and to determine the genetic purity of the genotypes. Furthermore, the wild genotypes may be useful in hybridization program with other cultivated commercial varieties or species to broaden the genetic base of mango genotypes (Mukherjee, 1997).

Based upon the pedigree and geographical origin, phenotypic approach is used to distribute genotypes into various groups (Schnell et al., 2005). However, this approach has limitation for grouping genotypes based on their genetic similarity. Therefore, an alternative Bayesian based cluster analysis approach is applied in this study by using STRUCTURE, which distributed the genotypes into three different gene pools based on allele information per locus. The cross pollination and out crossing may hold true for $M$. indica too, as the STRUCTURE analysis revealed some level of population admixture and gene exchange. Interestingly, this approach was able to distribute different genotypes based on several criteria like geographical origin and type (cultivated or wild). The highest likelihood was observed for $\mathrm{K}=3$, which separated the wild and cultivated mango genotypes into two different genetic backgrounds. The Pakistani genotypes were divided into two groups, which represents cultivated and wild separately, and showed two distinct genetic backgrounds. While, cluster of Indian varieties did not change and seem to be almost same. This analysis also confirmed that cultivated varieties of Pakistan share the genetic similarity with Indian origin, while wild genotypes from northern Punjab, which make cluster with cultivated varieties, have close relationship with them. The Indian mango genotypes have different genetic backgrounds and showed clear differences from other genotypes. Both 'Sufaid Chaunsa' and 'Kala Chaunsa', which were selected based on their superior fruit quality, are the variants of 'Samar Bahisht Chaunsa', whose origin is India (Rajwana et al., 2008). So, these 
genotypes showed a close genetic relationship with Indian germplasm. India is considered the primary center of origin for mango (Ravishankar et al., 2000). It is worth mentioning that both countries were united till 1947 and it is logical to conclude the similar genetic base for few Pakistani and Indian mango cultivars. But, Pakistani wild genotypes do not have Indian genetic background. So, Himalayan region of Pakistan might be center of diversity for mango germplasm in Pakistan. However, it can be confirmed by the further analysis of more wild genotypes from India and other mango growing countries.

The genetic relationship of Pakistani mango germplasm was determined by hierarchical clustering based on minimum variance ward method. Three major groups distinguished fifteen genotypes (34\%) originated from AJK and second group contained fifteen genotypes (18\%) from Northern Punjab with two cultivated varieties. The remaining cultivated varieties (47\%) showed narrow genetic basis, which could probably be due to ancient selection of cultivars from the existing cultivars of India. Rajwana et al. (2008) reported similar genetic relationship among cultivated Pakistani and Indian genotypes by RAPD markers. The factorial correspondence analysis also showed similar results which separated the mango genotypes in three groups. These findings are also in accordance with both of our results obtained by the dendrogram and Bayesian cluster analyses. The genetic exchange within species rather than ancient relationships has been emphasized to determine genetic structure or genetic diversity. Although, sometimes restriction occurs in structure exchange across the species range, either limited dispersion of pollen or seed, and by geographical distribution of the genotypes (Schaal et al., 1989). Since, the information on the precise migration of these wild mango plants from center of diversity is still unknown, it is most probable that the wild Pakistani mangoes are either descendants of isolated plants or relics of past migrants, which planted in the past but now have been established in the wild.

Conclusions: The reported results provide detailed information about the genetic makeup of new germplasm sources and genetic diversity in wild mango germplasm which could be useful to improve the yield and quality of existing varieties. Our results confirm that wild genotypes have high genetic diversity and unique structure pattern than the cultivated genotypes originated from Pakistan and India, which could be the result of the long history of mango cultivation in Pakistan. The results of the study clearly revealed that microsatellites can successfully be used to characterize mango germplasm of Pakistan.

Acknowledgements: The authors gratefully acknowledge Dr. Iqrar Ahmad Khan and Dr. Asif Ali for their kind help in undertaking this study and for helpful comments to prepare this manuscript. Mr. Rehan Riaz is thankful to Higher Education Commission of Pakistan for Indigenous PhD Fellowship (5000 Batch VII). This study was also supported by the mango project PARB-150. The authors also extend the gratitude to the owners of different mango orchards for the help during the sample collection.

\section{REFERENCES}

Amin, M. and M. Hanif. 2002. Cultivation of mango in Dera Ismail Khan. Agri. Res. Institute, Ratta, D.I. Khan, Pakistan. pp.1-18.

Ariffin, Z., M.S.M. Sah, S. Idris and N. Hashim. 2015. Genetic diversity of selected Mangifera species revealed by inter simple sequence repeats markers. Int. J. Biodiv. 15:1-8.

Azmat, M.A., A.A. Khan, I.A. Khan, I.A. Rajwana, H.M.N. Cheema and A.S. Khan. 2016. Morphological characterization and SSR based DNA fingerprinting of elite commercial mango cultivars. Pak. J. Agri. Sci. 53:321-330.

Azmat, M.A., I.A. Khan, H.M.N. Cheema, I.A. Rajwana, A.S. Khan and A.A. Khan. 2012. Extraction of DNA suitable for PCR applications from mature leaves of Mangifera indica L. J. Zhejiang Univ. Sci. 13:239-243.

Begum, H., M.T. Reddy, S. Malathi, B.P. Reddy, G. Narshimulu, J. Nagaraju and E.A. Siddiq. 2014. Morphological and microsatellite analysis of intravarietal heterogeneity in 'Beneshan' mango (Mangifera indica L.). Int. J. Agri. Food Res. 3:16-33.

Cunha, J., T. Santos and C. Carneiro. 2009. Portuguese traditional grapevine cultivars and wild vines (Vitis vinifera L.) share morphological and genetic traits. Genet. Resour. Crop Evol. 56:975-989.

Dillon, N.L., I.S.E. Bally, C.L. Wright, L. Hucks, D.J. Innes and R.G. Dietzgen. 2013. Genetic diversity of the Australian National Mango Genebank. Sci. Hortic. 150:213-226.

Duval, M.F., J. Bunel, C. Sitbon and A.M. Risterucci. 2005. Development of microsatellite markers for mango (Mangifera indica L). Mol. Ecol. Notes 5:824-826.

Evanno, G., S. Regnaut and J. Goudet. 2005. Detecting the number of clusters of individuals using the software STRUCTURE: a simulation study. Mol. Ecol. 14:26112620.

FAOSTAT. 2015. Food and Agriculture Organization of the United Nations database. Available online with updates at http://faostat.fao.org

Galvez-Lopez, D., M. Salvador-Figueroa, E.N. Becerra-Leor, M. Gonza'lezPaz, S. Herna'ndez-Delgado and N. Mayek-Perez. 2010. Molecular diversity and genetic relationships of mango germplasm from Chiapas, Mexico. Agrociencia 44:907-915. 
Hirano, R., H. Than and K.N. Watanabe. 2010. Myanmar mango landraces reveal genetic uniqueness over common cultivars from Florida, India, and Southeast Asia. Genome 53:321-330.

Honsho, C., K. Nishiyama, W. Eiadthong and K. Yonemori. 2005. Isolation and characterization of new microsatellite markers in mango (Mangifera indica). Mol. Ecol. Notes 5:152-154.

Karihaloo, J.L., Y.K. Dwivedi, S. Archak and A.B. Gaikwad. 2003. Analysis of genetic diversity of Indian mango cultivars using RAPD markers. J. Hort. Sci. Biotechnol. 78:285-289.

Khan, A.S., S. Ali and I.A. Khan. 2015. Morphological and molecular characterization and evaluation of mango germplasm: An overview. Sci. Hortic. 194:353-366.

Khan, I.A., A.S. Khan, I.A. Rajwana, A.A. Khan, M.A. Azmat and S.A. Raza. 2016. Premium quality mango genotypes for extended harvest season. HortScience 51:1609-1612.

Luo, C., X. He, H. Chen, Y. Hu, S. Ou and M.P. Gao. 2010. Analysis of diversity and relationships among mango cultivars using Start Codon Targeted (SCoT) markers. Biochem. Syst. Ecol. 38:1176-1184.

Mukherjee, S.K. 1997. Introduction, botany and importance. In: R.E. Litz (ed.), The Mango: Botany, Production and Uses. CAB International, Wallingford, UK. pp.1-19.

Nafees, M., S. Ahmad, R. Anwar, I. Ahmad, Maryyam and R.R. Hussnain. 2013. Improved horticultural practices against leaf wilting, root rot and nutrient uptake in mango (Mangifera indica L). Pak. J. Agri. Sci. 50:393-398.

Peakall, R. and P.E. Smouse. 2012. GenALEx 6.5: genetic analysis in Excel. Population genetic software for teaching and research-an update. Bioinformatics 28:2537-2539.

Perrier, X., A. Flori and F. Bonnot. 2006. Data analysis methods. In: P. Hamon, M. Seguin, X. Perrier, J.C. Glaszmann, P. Hamon and M. Seguin (eds.), Genetic Diversity of Cultivated Tropical Plants. Science Publishers, Montpellier, France. pp.43-76.

Pritchard, J.K., M. Stephens and P. Donnelly. 2000. Inference of population structure using multilocus genotype data. Genetics 155:945-959.

Rahman, M.L., M.G. Rabbani, M.N.A. Siddique, M.A. Rahman, E.J. Garvey and E.H.M.S. Rahaman. 2007. Molecular characterization of 28 mango germplasm using RAPD. Plant Tissue Cult. Biotechnol. 17:71-77.

Rajwana, I.A., N. Tabbasam, A.U. Malik, S.A. Malik, M. Rahman and Y. Zafar. 2008. Assessment of genetic diversity among mango (Mangifera indica L) genotypes using RAPD markers. Sci. Hortic. 117:297-301.

Rajwana, I.A., A.U. Malik, A.S. Khan, B.A. Saleem and S.A. Malik. 2010. A new mango hybrid shows better shelf life and fruit quality. Pak. J. Bot. 42:2503-2512.
Rajwana, I.A., I.A. Khan, A.U. Malik, B.A. Saleem, A.S. Khan, K. Ziaf, R. Anwar and M. Amin. 2011. Morphological and biochemical markers for varietal characterization and quality assessment of potential indigenous mango (Mangifera indica) germplasm. Int. J. Agric. Biol. 13:151-158.

Ravishankar, K.V., L. Anand and M.R. Dinesh. 2000. Assessment of genetic relatedness among mango cultivars of India using RAPD markers. J. Hort. Sci. Biotechnol. 75:198-201.

Ravishankar, K.V., B.H.R. Mani, L. Anand and M.R. Dinesh. 2011. Development of new microsatellite markers from mango (Mangifera indica) and cross-species amplification. Amer. J. Bot. 98:96-99.

Ravishankar, K.V., P. Bommisetty, A. Bajpai, N. Srivastava, B.H. Mani, C. Vasugi, S. Rajan and M.R. Dinesh. 2015. Genetic diversity and population structure analysis of mango (Mangifera indica) cultivars assessed by microsatellite markers. Trees 29:775-783.

Samal, K.C., R.C. Jena, S.S. Swain, B.K. Das and P.K. Chand. 2012. Evaluation of genetic diversity among commercial cultivars, hybrids and local mango (Mangifera indica L) genotypes of India using cumulative RAPD and ISSR markers. Euphytica 185:195-213.

Schaal, B.A., D.A. Hayworth, K.M. Olsen, J.T. Rauscher and W.A. Smith. 1989. Phylo-geographic studies in plants: problems and prospects. Mol. Ecol. 7:465-474.

Schnell, R.J., C.T. Olano, W.E. Quintanilla and A.W. Meerow. 2005. Isolation and characterization of 15 microsatellite loci from mango (Mangifera indica L) and cross-species amplification in closely related taxa. Mol. Ecol. Notes 5:625-627.

Schnell, R.J., J.S. Brown, C.T. Olano, A.W. Meerow, R.J. Campbell and D.N. Kuhn. 2006. Mango genetic diversity analysis and pedigree inferences for Florida cultivars using microsatellite markers. J. Amer. Soc. Hort. Sci. 13:214-224.

Singh, K.K. and J.S. Jawandha. 1962. Some promising seedling mango trees of Punjab. Punjab Hort. J. 2:139142.

Singh, S., A.B. Gaikwad and J.L. Karihaloo. 2009. Morphological and molecular analysis of intra cultivar variation in Indian mango (Mangifera indica L) cultivars. Acta Hort. 829:205-212.

Souza, I.G.B., S.E.S. Valente, F.B. Britto, V.A.B. de Souza and P.S.C. Lima. 2011. RAPD analysis of the genetic diversity of mango (Mangifera indica) germplasm in Brazil. Genet. Mol. Res. 10:3080-3089.

Surapaneni, M., L.R. Vemireddy, H. Begum, B.P. Reddy, C. Neetasri, J. Nagaraju, S.Y. Anwar and E.A. Siddiq. 2013. Population structure and genetic analysis of different utility types of mango (Mangifera indica L) germplasm 
of Andhra Pradesh state of India using microsatellite markers. Plant Syst. Evol. 299:1215-1229.

Tomar, R.S., H.P. Gajera, R.R. Viradiya, S.V. Patel and B.A. Golakiya. 2011. Phylogenetic relationship among mango (Mangifera indica L) landraces of Saurashtra based on DNA fingerprinting. J. Hort. For. 3:379-38.

Ullah, H., S. Ahmad, M. Amjad and M.A. Khan. 2012. Response of mango cultivars to modified atmosphere storage at an ambient temperature cv. Alphanso and Chounsa. Pak. J. Agri. Sci. 49:323-329.

Viruel, M.A., P. Escribano, M. Barbieri, M. Ferri and J.I. Hormaza. 2005. Fingerprinting, embryo type and geographic differentiation in mango (Mangifera indica L, Anacardiaceae) with microsatellites. Mol. Breed. 15:383-393.

Yamanaka, N., M. Hasran, D.H. Xu, H. Tsunematsu, S. Idris and T. Ban. 2006. Genetic relationship and diversity of four Mangifera species revealed through AFLP analysis. Genet. Res. Crop Evol. 53:949-954.

Zaied, N.S., S.A.A. Khafagy and M.A. Saleh. 2007. Evaluation of some mango species by fruit characters and fingerprint. Res. J. Agric. Biol. Sci. 3:316-320. 